\title{
Time to trap an ytterbium ion
}

Pauline Rigby

A single ion held motionless in an electrodynamic trap may provide the ultimate standard for measuring time'. The current standard is defined in terms of the energy separation between two hyperfine energy levels in the ground state of a caesium atom (Fig. 1). At the moment, the best caesium clocks $s^{2,3}$ are accurate to within three parts in $10^{15}$, but the detection of a new atomic transition at the UK National Physical Laboratory, reported in Physical Review Letters ${ }^{4}$ this month, sets the scene for an optical clock more than 1,000 times as accurate. Such high accuracy is equivalent to an error of less than one second in measuring the age of the Universe.

When an electron moves between two energy levels of an ion, it absorbs or ejects a photon whose energy matches the difference between the two levels. To be a suitable candidate for an optical frequency standard, the ion must possess a long-lived or metastable level, and the photon wavelength produced by the transition between this metastable level and the ground state must be accessible to continuous-wave lasers. The rare-earth metal ytterbium contains three potentially suitable transitions: at 411,435 and 467 nanometres. The first two of these have already been observed ${ }^{5,6}$, but the third has eluded detection until now. But it is precisely because it is so difficult to observe that the transition is so interesting.

Roberts et al. ${ }^{4}$ have seen the transition for the first time, and found that the upper state lives for about ten years. This is the time it takes for an electron to fall spontaneously back to the ground state, a phenomenon called fluorescence. If we know the lifetime

\section{IMAGE UNAVAILABLE FOR COPYRIGHT REASONS}

Figure 1 Old timer. This is the first caesium atomic clock, developed in 1955. A new standard may be set by single-ion ytterbium resonators.

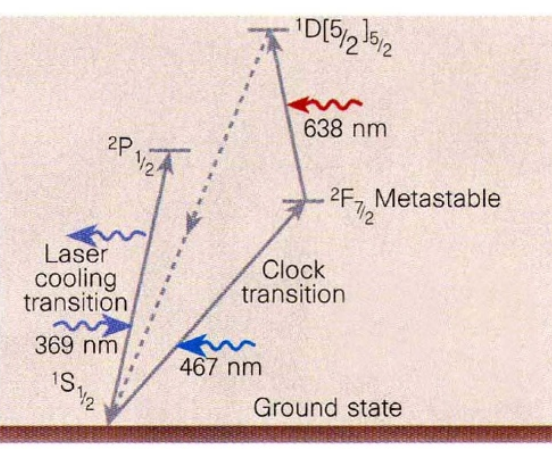

Figure 2 Simplified representation of energy levels in an ytterbium ion. An electron is cooled with ultraviolet light, driven into the metastable level with blue light and finally recycled with red light.

of the state, then its linewidth — the spread of energies about a central frequency - is fixed by Heisenberg's uncertainty principle. The more familiar form of the principle says that the more accurately the position of a particle is determined, the more uncertain we must be about its momentum. Another form of this law relates time and energy. The product of the two variables, position and momentum or lifetime and linewidth, has a fixed value. Because the lifetime of the 467 $\mathrm{nm}$ transition is so long, it is extremely narrow in frequency - perhaps the narrowest spectral line available.

The key parameter for the stability of a clock is its $Q$ factor, which is the ratio of the transition frequency to the linewidth, or the proportional uncertainty in the frequency of a single emitted photon. For an ion under normal atmospheric conditions there are many mechanisms that will broaden the linewidth, such as Doppler (motion induced) broadening. But the Doppler effect can be exploited to eliminate itself and bring the ion to rest inside a trap, by the process of laser cooling. A laser with a wavelength slightly off the strong 369 $\mathrm{nm}$ transition is used. Only when the ion is moving into the laser beam (as it vibrates) does the laser frequency appear to match the transition and a photon can be absorbed. When the ion fluoresces, on average it releases a slightly more energetic photon than the one it received, so that over time the ion's vibrational energy is gradually depleted.

Once the ion is cooled down to a few millikelvin and trapped in a space whose dimensions are smaller than the wavelength of the laser light, then the frequency of the clock transition can be located. The region around the transition is scanned in small steps with a second laser beam which has a similarly narrow linewidth (otherwise it is like trying to pick up a pin with boxing gloves on). To scan a wide region about the clock transition with enough care to be sure they hadn't missed it would have taken several years, so the group first narrowed down the search region, from $2.6 \mathrm{GHz}$ to $8 \mathrm{MHz}$, by measuring two other connected transitions and subtracting the frequencies ${ }^{5,7}$.

Fluorescence from the laser cooling transition is used to indicate when a $467 \mathrm{~nm}$ photon has been absorbed. A $369 \mathrm{~nm}$ laser and the scanning probe laser illuminate the sample alternately. The electron continually cycles up and down the $369 \mathrm{~nm}$ transition, fluorescing each time it falls back to the ground state, until the $467 \mathrm{~nm}$ transition gets resonantly excited by the probe laser (Fig. 2). Then the fluorescence stops because the electron gets stuck in the long-lived state.

Now the problem is how to return the ion to its ground state so that the scan can continue. One option would be to wait ten years, but this is obviously impractical. A third laser beam is used to send the electron on up to a higher energy state with a short lifetime. It quickly falls back to the ground state from there, and the process can begin again.

Initially, the number of transitions observed was only one per hour, but this has now been increased to two per minute which has made it possible to build up a spectral profile of the clock transition and measure its frequency to better than $1 \mathrm{MHz}$, not far off one part in a billion. This is the first time that such a weak, so-called 'octupole' transition has been driven.

There is still a lot of work to be done before they can make a clock. The rate of jumps must be increased, by using a laser with an even narrower linewidth to locate the clock transition more precisely. The National Physical Laboratory is also working on more accurate methods for down-converting trapped ion optical frequencies to microwave frequencies for comparison with the caesium standard there.

Why do we need better clocks? No doubt physicists will rub their hands with glee at the prospect of devising new experiments to test the theory of relativity. Cold, trapped ions also present the ideal medium for finding out whether the fundamental physical constants, such as Planck's constant, really are constant. Satellite-based global positioning systems and long-baseline interferometry in radio telescopes are among the many applications that will benefit from the advance in accuracy of measuring something as fundamental as time.

Pauline Rigby is in the Department of Engineering Science, University of Oxford, Parks Road, Oxford OX1 3PJ, UK.

\footnotetext{
Dehmelt, H. G. IEEE Trans. Instrum. Meas. 31, 83-87 (1982).

2. Gibble, K. \& Chu, S. Metrologia 29, 201-212 (1992).

3. Clairon, A et al. in Proc 5 th Symp. Frequency Standards Metrology (ed. Bergquist, J. C.) 49-59 (World Scientific, Singapore, 1996).

4. Roberts, M. et al. Phys. Rev. Lett. 78, 1876-1879 (1997).

. Gill, P. et at. Phys. Rev. A 52, 909-912 (1995).

6. Engelke, D. \& Tamm, C. Europhys. Lett. 33, 347-352 (1996)
}

Bell, A. S. et al. J. Mod. Opt. 39, 381-387 (1992). 